\title{
Allégorie et déconstruction dans Le Roman de la rose
}

Carine BOURGET

T a complexité de la double structure allégorique du Roman de la rose a donné cours à diverses interprétations. ${ }^{1}$ Parmi les problèmes soulevés par les critiques à ce sujet, deux points se détachent. Le premier est le manque de consensus quant à la définition de l'allégorie. La personnification est-elle une allégorie? Peut-on lire le niveau littéral avec le niveau figuré ou ce dernier exclut-il le premier? La deuxième source de divergence concerne l'unité d'une oeuvre écrite par deux auteurs à quarante ans d'intervalle l'un de l'autre. La plupart des critiques voient une nette différence entre les deux parties de l'oeuvre; selon eux, la première, composée par Guillaume de Lorris, dépeint les valeurs idéales de l'amour courtois, tandis que la continuation de Jean de Meun s'oppose aux théories de son prédécesseur, car elles ne sont que prétexte à discourir sur les sujets les plus diverses.

Cependant, deux critiques ont proposé une interprétation unificatrice de l'oeuvre. Selon Alan Gunn, le sujet du Roman de la rose ne cesse à aucun moment d'être en rapport avec l'amour dans 
son sens le plus large (Gunn 484); et pour Robertson, l'ensemble est une allégorie de la chute de l'homme du Paradis et ses conséquences (Arden 51). A la même recherche d'une idée qui lie les deux parties, cet essai propose d'interpréter le Roman de la rose comme une allégorie de la quête de la vérité. Sous cette perspective, l'analyse de passages dans lesquels la voix du narrateur'2 se fait entendre nous permet de montrer que Guillaume de Lorris et Jean de Meun traitaient le même problème. Nous verrons que les deux parties s'accordent à présenter cette quête comme étant irréalisable, mais que cette impossibilité est donnée sous deux modes différents: ironique chez Guillaume de Lorris, et parodique chez Jean de Meun.

Plusieurs critiques ont signalé que la quête de la vérité est souvent représentée de façon allégorique par la possession sexuelle de la femme par l'homme. Jonathan Culler glose Derrida ainsi:

Celebrations of woman or the identification of woman with some powerful force or idea -truth as a woman, liberty as a woman, the muses as womenidentify actual women as marginal. Woman can be a symbol of truth only if one presumes that those seeking truth are men. (167)

Dans Spéculum de l'autre femme, Luce Irigaray démontre que la subjectivité dépend de l'opposition entre le sujet et l'objet, et que dans une culture patriarcale, la femme occupe toujours le rôle de l'objet contre lequel l'homme se définit en tant que sujet:

Subjectivity denied to woman: indisputably this provides the financial backing for every irreducible constitution as an object: of representation, of discourse, of desire. (133) ${ }^{3}$

La femme objectifiée devient image de l'objet dans un sens général, et en particulier du savoir absolu ou de la vérité:

Dispersing, piercing those metaphors - particularly the photological ones which have constituted truth by the premises of Western philosophy: virgin, dumb and veiled in her nakedness... (Irigaray 136) 
La réduction de la femme à l'état d'objet se retrouve dans les deux parties du Roman de la rose.

L'objet signifié par l'allégorie de la rose est donné de façon explicite dès les premiers vers de Guillaume de Lorris:

C'est cele qui tant a de pris

Et tant est digne d'estre amee

Qu'ele doit estre rose clamee.

(Vv. 42-45: C'est celle qui a tant de prix et qui est à ce point digne d'être aimée, qu'on doit l'appeler "rose.") 4

C'est la femme aimée qui est représentée par une fleur, et tout ce qui pourrait constituer sa personnalité est soigneusement détaché d'elle et fragmenté en diverses personnifications, telles Bel Accueil, Chasteté, Danger, pour n'en citer que quelques-unes. Ces personnifications ne sont que des projections des pensées et des désirs que la société patriarcale et les fantasmes du narrateur attribuent à la femme. 5

Jean de Meun conserve la même allégorie de la rose représentant la femme, comme l'indiquent les nombreuses métaphores de pénétration sexuelle. Avant d'atteindre la rose, l'amant introduit son bourdon dans la meurtrière, et pour ce faire il doit briser une palissade, puis cheminer dans un chemin étroit (Vv. 21640-21677). Le mot "reliques"(v. 25589) qui se réfère à l'organe sexuel de la femme, évoque le discours de Raison à l'amant, dans lequel elle affirme que le lien entre la chose et le mot est purement conventionnel (voir les vers 6941-7187), et dans lequel le mot "reliques" était déjà associé à un organe sexuel ("coilles"), bien que cette fois masculin. La double allégorie de la cueillette-pèlerinage qui termine le roman est le récit de l'union sexuelle de l'amant et de sa bien-aimée. La rose sera, du début à la fin du roman, un objet de convoitise passif, ce qui permet de relire sa cueillette comme une allégorie de la recherche de la vérité.

Dans les deux parties, le désir de posséder la rose motive la narration, et ce désir est entretenu par la distance qui sépare la rose de l'amant. La distance, nécessaire pour susciter le désir, est 
présente non seulement dans le thème, mais aussi dans la structure du Roman de la rose, car elle est à la base de la figure de rhétorique de l'allégorie que Paul de Man définit comme:

a relationship between signs in which the reference to their respective meanings has become of secondary importance. But this relationship between signs necessarily contains a constitutive temporal element; it remains necessary, if there is to be allegory, that the allegorical sign refer to another sign that precedes it. The meaning constituted by the allegorical sign can then consist only in the repetition ... of a previous sign with which it can never coincide ... allegory designates primarily a distance in relation to its own origin. (190-1)

De Man apporte une nouvelle perspective sur la figure de l'allégorie, car il n'est plus question de privilégier l'un des niveaux littéral ou figuré au détriment de l'autre. Cette définition met en relief l'inexorabilité de la distance entre le signifiant (la rose) et le signifié (la femme aimée) qui constituent l'allégorie du Roman de la rose.

La distance entre l'amant et l'objet de ses désirs est donnée, avant même ses mésaventures pour essayer de cueillir la rose et au cours desquelles les obstacles seront multipliés, comme infranchissable. Le début du récit laisse prévoir que toute tentative de contact entre eux est vouée à l'échec. L'amant de Guillaume découvre les rosiers dans le reflet du cristal de la fontaine de Narcisse, et l'image du jardin qu'il contemple est médiatisée par le miroir qui se révélera trompeur par la suite:

Cil mireors m'a deceü:

Se j'eusse avant queneü

Quel la force ere et sa vertu,

Ne m'i fusse ja embatu

(Vv. 1606-1609: Ce miroir m'a trompé: si j'avais su d'avance quelle était sa force et son pouvoir, jamais je ne me serais précipité sur elle). 
Contrairement au mythe de Narcisse, ce n'est pas l'eau de la fontaine qui sert de miroir ici; au contraire, sa limpidité laisse voir le cristal qui gît au fond de l'eau et qui lui renvoie l'image (en deux moitiés) du verger. L'illusion de l'image contemplée dans le miroir n'est qu'un reflet illusoire de la réalité. La fragmentation de ce reflet présage l'impossibilité d'unifier la réalité et la perception que nous en avons, car la médiatisation du cristal semble prévenir tout contact direct et immédiat avec la rose.

Le signifié donné (c'est-à-dire la femme) n'est lui-même qu'un signifiant pour un signifié que les deux auteurs promettent de dévoiler à plusieurs reprises (sans pour autant le faire). Guillaume promet à deux reprises de donner une explication, sans jamais tenir ses promesses, ou plutôt en différant indéfiniment le moment annoncé:

Mes james n'orrez meus descrivre

La verite de la matiere,

Quant j'auré espont le mistere.

(Vv.. 1597-1599: jamais vous n'entendrez mieux exposer la vérité de la matière que lorsque j'aurai explicité le mystère)

Et la vos dirai sanz grevance;

La verite qui est coverte

Vos en sera lors toute aperte,

Quant espondre m'orroiz le songe,

Car il n'i a mot de mensonge.

(Vv. 2070-2071: je vous la dirai sans peine; sa vérité qui est voilée vous sera dévoilée tout entière au moment où vous m'entendrez expliciter le songe,

car il ne contient un mot de mensonge)

Cette prolifération de signifiants rend impossible toute tentative pour cerner le signifié, car la rose représente la femme, qui à son tour réfère à autre chose, et cette donnée inconnue est, d'après le poète, cachée sous le songe.

Le narrateur présente le rêve qu'il s'apprête à conter comme 
prémonitoire. ${ }^{6}$ Guillaume de Lorris se conforme ainsi à la tradition médiévale du songe prophétique porteur de vérité. Cependant, Poirion remarque pertinemment que la signification s'exerce donc aux deux niveaux du songe et de l'allégorie: "le signifié apparent du récit doit être traité comme le signifiant d'un autre signifié encore caché, et que l'inachèvement de l'oeuvre, ou l'artifice de l'auteur, laisseront à découvrir" ( 167). Selon notre interprétation, le savoir absolu se superpose à la femme, elle-même étant déjà superposée à la rose.

De plus, l'accès à cette vérité promise par le songe (premier intermédiaire) est de nouveau différé par l'allégorie (second intermédiaire), dont le sens ne sera jamais révélé. La distance inhérente à toute allégorie est multipliée par deux par l'intermédiaire de cette double allégorie: ainsi, la vérité est déguisée sous le songe, et le songe lui-même est sous la forme d'une allégorie qui équivaut à l'équation suivante: la rose=la femme=?; l'une des données reste inconnue à jamais dans le texte même, et toute proposition (comme la nôtre qui interprète l'élément manquant comme la vérité absolue) ne peut être que spéculation du lecteur. Ces deux intermédiaires dont la fonction est de garantir l'aspect véridique du récit ne font qu'obscurcir et retarder le sens final annoncé, qui est sans cesse différé. Nous pouvons récrire notre équation de la façon suivante: la vérité $=$ le songe $=$ l'allégorie $=$ la rose $=$ la femme $=$ la vérité. Ce cercle fermé sur lui-même représente le récit du Roman de la rose, quête impossible de la vérité parce qu'elle se fait toujours par intermédiaire.

La question qui continue à préoccuper les critiques (et à laquelle on ne pourra jamais répondre que de facon spéculative) concerne la fin en suspens de la première partie: était-ce dû à un abandon volontaire ou à une interruption accidentelle, comme l'indique son successeur? Contrairement à l'affirmation suivante: "le signe allégorique, chargé de découvrir la vérité chez Guillaume de Lorris, est dénoncé comme procédé ludique par Jean de Meun" (Poirion 181), les réitérations montrent que tout comme Jean de Meun, Guillaume de Lorris a "toujours déjà" (pour emprunter le 
terme de Derrida) renoncé à la possibilité de donner un sens clos et fini à son allégorie. Que le poème commencé par Guillaume de Lorris ait été achevé ou non importe peu, car la tâche entreprise est condamnée à être prolongée de manière infinie.

Jean de Meun reprend cette tactique de promettre une explication$^{7}$ qui ne viendra jamais, bien que lui apporte une clôture expéditive au roman après des milliers de vers de discours. Si les sentiments amoureux de l'idéal courtois semblent au centre de la première partie, la deuxième en revanche porte son attention sur la sexualité (qui est associée à plusieurs reprises avec l'écriture, et par extension, avec le savoir). Après de longs discours qui diffèrent le moment final et dans lesquels Raison, Nature, Génius et la Vieille donnent des vues différentes de l'amour, Jean de Meun écrit la scène prévisible de la cueillette, mais de manière inattendue. La différence entre les deux parties du Roman de la rose ne réside pas tant dans le contenu que dans le ton, explicitement parodique de Jean de Meun, et plus ambigu de Guillaume de Lorris.

Heather Arden pose le problème de la position de Guillaume de Lorris de la façon suivante:

a scholar must decide, when describing Guillaume's portrayal of courtly values, whether the poet admiringly accepted those values or ironically questioned their validity. Irony, though, which is often the result of tone or suggestion, is a difficult matter for scholars to agree on. (93)

De Man a montré que le point commun entre l'allégorie et l'ironie, qui consiste à dire une chose en voulant en dire une autre, est une relation de discontinuité entre le sens et le signe. Selon lui,

the act of irony reveals the existence of a temporality that is definitely not organic, in that it relates to its source only in terms of distance and difference and allows for no end, for no totality. (203)

$\mathrm{Si}$ "the ironist invents a form of himself that is 'mad' but that does not know its own madness; he then continues to reflect on his own madness thus objectified" (de Man 198), alors l'amant est une pro- 
jection de Guillaume de Lorris, le premier ne sachant pas que sa quête ne pourra jamais aboutir. La folie de l'amant est donc de croire pouvoir atteindre son but (la rose/vérité) et l'auteur lui fait vivre ces aventures successives, tout en sachant qu'elles ne peuvent finir. Les obstacles se multiplient, et seule la suspension (temporaire) du récit les arrête.

Le passage de la cueillette de la rose, qui décrit clairement l'acte sexuel, peut être mis sous un nouveau jour en le comparant à l'allégorie de la caverne de Platon telle qu'Irigaray l'analyse, c'està-dire en lisant la caverne comme une métaphore de l'utérus (243). En associant l'accession à la vérité à la sortie de la caverne peuplée d'ombres, Platon dénigre le féminin. Dans la seconde partie de Spéculum, Irigaray

...takes Plato's parable of the cave, with its contrast between a maternal womb and a divine paternal logos, as the point of departure for a demonstration that philosophical categories have been developed to relegate the feminine to a position of subordination and to reduce the radical Otherness of woman to a specular relation... (Culler 58)

En multipliant les images de pénétration, Jean de Meun suggère un retour vers l'utérus-caverne. La possession de la rose, qui symbolisait l'impossible accession à la vérité chez Guillaume de Lorris, est doublement démystifiée par Jean de Meun, avec qui elle n'aboutit qu'à un retour à l'ignorance. Ainsi, la quête de la vérité en tant qu'adéquation entre la réalité et l'homme qui la pense est donnée comme impossible dans la totalité du récit, car la connaissance du monde par l'homme ne peut se faire que par la médiation du langage.

L'inachèvement du récit ironique de Guillaume est paradoxalement la seule fin envisageable de cette quête de la vérité allégorisée par la femme aimée. Jean de Meun parodie la prétention d'accéder à la vérité en caricaturant la quête de l'amant, et nous avons vu que Guillaume lui-même avait donné le but de son personnage comme étant impossible à atteindre. Selon Arden, Jean de 
Meun "is suggesting that the individual forms his or her judgment of truth through lived experiences" (111). Il se fait donc l'avocat de l'expérience, en parodiant la quête de l'amant, car tout ce que l'homme peut prétendre atteindre est un savoir relatif alors qu'il cherche l'absolu. 


\section{Notes}

1 Pour un excellent résumé historique et critique de la recherche publiée sur le Roman de la rose, voir Heather Arden, particulièrement les chapitres trois ("Allegory and Personification in the Rose)" et cinq ("Critical History of the Roman de la rose").

2 Nous espérons ainsi éviter l'écueil que beaucoup de critiques n'ont pu éviter et qui est le suivant: "The problem is whether these personifications are speaking for the author; many scholars have made this assumption, even pulling sections out of conflicting speeches to prove "the author's point of view" (Arden 54).

3 N'ayant à notre disposition que la traduction en anglais, nous nous excusons auprès du lecteur de ne pas citer le texte original.

4 Toutes les citations (langue originale et traduction) du Roman de la rose sont tirées de l'édition d'après les manuscrits BN 12786 et BN 378 traduite et annotée par Armand Strobel.

5 Pour ne citer qu'un exemple, Bel Accueil n'est qu'une projection des désirs de l'amant. A la fin, l'amant dit de lui “qu'il consentait et souffrait que je fisse tout ce qu'il savait devoir me plaire. Il invoqua pourtant un engagement que $\mathrm{j}$ 'avais pris et me déclara que j'agissais mal envers lui et que j'abusais. Mais il ne mit aucun obstacle" (1241). Selon les fantasmes du narrateur, la femme-rose ne vit que pour satisfaire ses moindres désirs, bien qu'ils soient contraires à la propre volonté ou l'intérêt de celle-ci.

6 Le récit de la première partie s'ouvre sur la constatation suivante:

Maintes genz cuident qu'en songe

N'ait se fable non et mençonge.

Mais on puet tel songe songier

Qui ne sont mie mençongier,

Ainz sont aprés bien aparant.

(Vv. 1-5: Nombreux sont ceux qui s'imaginent que dans les rêves il n'y a que fables et mensonges. Pourtant, il est possible de faire des rêves qui ne soient pas mensongers, mais qui par la suite se véri- 


\section{ALLEGORIE ET DECONSTRUCTION}

fient tout à fait).

7 "Bien orrez que ce senefie / Ainz que ceste oevre soit fenie" (Vv. 21217-21218: Vous entendrez bien ce que cela signifie avant que cette oeuvre ne soit finie).

Michigan State University 


\section{Ouvrages cités}

Arden, Heather M. The Romance of the Rose. Boston: Twayne Publishers, 1987.

Culler, Jonathan. On Deconstruction: Theory and Criticism after Structuralism. Ithaca, NY: Cornell UP, 1982.

De Man, Paul. "The Rhetoric of Temporality." Interpretation:

Theory and Practice. Charles S. Singleton, ed. Baltimore: Johns Hopkins UP, 1969: 173-209.

Gunn, Alan. The Mirror of Love: A Reinterpretation of "The Romance of the Rose." Lubbock, Texas: Texas Tech Press, 1952.

Irigaray, Luce. Speculum of the Other Woman. Trad. Gillian C. Gill. [1974]. Ithaca, NY: Cornell UP, 1985.

Lorris, Guillaume de et Jean de Meun. Le Roman de la rose. Armand Strubel, ed. et trad. Collection Lettres gothiques. Paris: Librairie Générale Française, 1992.

Poirion, Daniel. "De la signification selon Jean de Meun." Archéologie du signe. Brind'Amour Lucie et Eugene Vance, eds. Toronto, Canada: Institut Pontifical d'Etudes Médiévales, 1983: 165-185. 

